\title{
Stability Analysis of Wheat Populations and Mixtures Based on the Physical, Compositional and Processing Properties of the Seeds
}

\author{
K. Tremmel-Bede ${ }^{1}$, P. Mikó ${ }^{1 *}$, M. Megyeri ${ }^{1}$, G. Kovács $^{1 \dagger}$, S. Howlett ${ }^{2}$, B. Pearce ${ }^{2}$,

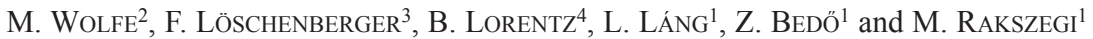 \\ ${ }^{1}$ Agricultural Institute, Centre for Agricultural Research, Hungarian Academy of Sciences, Brunszvik u. 2, \\ H-2462 Martonvásár, Hungary \\ ${ }^{2}$ The Organic Research Centre, Elm Farm, Hamstead Marshall, Newbury, Berkshire, RG20 0HR, UK \\ ${ }^{3}$ Saatzucht Donau GmbH \& Co KG, Saatzuchtstrasse 11, 2301 Probstdorf, Austria \\ ${ }^{4}$ INRA - UMR Diversité et Génome des Plantes Cultivées - Domaine de Melgueil - 34130 Mauguio, France
}

(Received 14 October 2015; Accepted 21 March 2016;

Communicated by A. Goyal and T. Harangozó)

Six cropping populations, three variety mixtures and one diversity population were developed from winter wheat varieties and studied for physical, compositional and end-use quality traits for three years (2011-2013) under different European climatic and management conditions in order to study the stability of these traits resulted by the genetic diversity. The beneficial compositional and nutritional properties of the populations were assessed, while variation and stability of the traits were analysed statistically. No significant differences were found among the populations in low-input and organic management farming systems in the physical, compositional and processing properties, but there was a difference in the stability of these traits. Most of the populations showed higher stability than the control wheat variety, and populations developed earlier had higher stability than those developed later. Furthermore, some populations were found to be especially unstable for some traits at certain sites (mostly at Austrian, Swiss and UK organic sites). Protein content of the populations was high (13.0-14.7\%) without significant difference among them, but there was significant variation in their gluten content (28-36\%) and arabinoxylan content (14.6-20.3 mg/g). The most outstanding population for both protein and arabinoxylan content was a Hungarian cropping population named ELIT-CCP. It was concluded that the diversity found in the mixtures and CCPs have stabilizing effect on the quality parameters, but a higher stability was observed under low-input than under organic conditions. These results could be beneficial not only for breeders but also for the consumers in the long run.

Keywords: wheat, organic, stability, CCP, GGE biplot, dietary fibre

Abbreviations: AX - Arabinoxylan; CCP - Composite cross-population; CV Coefficient of variation; DF - Dietary fibre; GI - Gluten index; HI - Hardness index; LI Low input (refers to low input conventional field); O - Organic (refers to organic field); TKW - Thousand-kernel weight; TOT - Total; TW - Test weight; WE - Water extractable

\footnotetext{
*Corresponding author; E-mail: miko.peter@agrar.mta.hu; Phone: +3622569-500; Fax: +3622460-213
} 


\section{Introduction}

Many studies (e.g. Heal et al. 2004; Murphy et al. 2005; Phillips and Wolfe 2005; Finckh 2008; Hajjar et al. 2008; Wolfe et al. 2008; Newton et al. 2009; Lammerts van Bueren et al. 2010) have highlighted the need for increased biodiversity in the field as it is essential to improve sustainability in agricultural systems and in food and nutrition security (Frison et al. 2011). The need for greater biodiversity is especially strong in organic and 'lowinput' systems due to the increased heterogeneity of these environments in time and space (Altieri 1999; Ceccarelli et al. 2007; Finckh 2008; Wolfe et al. 2008; Østergård et al. 2009). Organic farming is more than merely replacing chemical pesticides and fertilizers with organic ones. Its goal is to stimulate a high level of self-regulation in the system through functional diversity in and above the soil, rather than depending on external inputs (Østergård et al. 2009). Therefore, factors like cultivar selection are more critical for organic than for conventional farmers. Organic growers are largely dependent on cultivars bred for conventional systems because there is a lack of breeding specifically for organic systems and thus conventional cultivars dominate the available commercial choices. However, only few of these are optimal for organic farming systems because traits associated with independence from external inputs have not received high priority in current breeding programs (Lammerts van Bueren and Myers 2012). Since more than a decade plant breeders have been producing genetically diverse and heterogeneous materials targeting the organic cultivation conditions, but only some of them are commercially available. There are a number of ways to deliver this heterogeneity needed by organic farmers. One method is the creation of variety mixtures, where the components of the mixture could be landraces, heritage varieties or modern varieties that are grown and harvested together. An alternative approach to produce greater genetic diversity is to create composite cross-populations (CCP). These are developed by crossing a group of diverse parents, then growing and harvesting the subsequent generations as a bulk population (Suneson 1956; Döring et al. 2015). It is already known that CCPs and variety mixtures have several physiological benefits when compared to less genetically diverse varieties. These include vigorous early growth, better weed suppression, and improved nutrient use efficiency in the early growing phase, and resistance against pests and soil bacteria (Wolfe et al. 2008). However, there is little evidence about whether these mixtures and populations have any benefits either from a nutritional or a processing point of view for local or global food market.

Therefore several CCPs and variety mixtures were investigated in the current study in order to assess their stability regarding the physical, compositional and breadmaking quality traits and to identify their beneficial, compositional and nutritional properties.

\section{Materials and Methods}

\section{Plant material}

In order to develop competitive wheat materials for organic and low-input conditions with good performance, six cropping populations were produced in Austria (A), Hungary 
(H) and the UK (UK) (POP-AT (A), English-Composite (H, ENG-CCP), Elite-Composite (H, ELIT-CCP), Hungarian-Composite (H, HUN-CCP), YQ-CCP (UK) and NIAB-EliteCCP (UK, NIAB-CCP)). These populations were studied together with three variety mixtures (MIX-AT (A), YQ-MIX (UK), NIAB-Elite-MIX (UK, NIAB-MIX)) and one diversity population from France (INRA-60parent-CCP called INRA-CCP in this paper). Development of YQ-CCP and YQ-MIX was explained in detail by Döring et al. (2015). One winter wheat (Triticum aestivum L.) variety, Mv-Emese $(\mathrm{H})$ was used as a 'common' control in all locations. Mv-Emese is a hard red winter wheat variety with high gluten content and excellent breadmaking quality (its Farinograph and Alveograph values are excellent). It is an early maturing genotype which has good tolerance against environmental stresses. The years and sites of the study for each population are listed in Table S1*.

\section{Plant origin}

Development strategy and parental variety selection could basically determine the processing quality and performance of CCPs/mixtures. YQ-CCP and YQ-MIX were developed in the UK using nine high-yielding and twelve high-quality wheat genotypes based on 190 crosses between them (Döring et al. 2015). Similarly, NIAB-CCP and NIAB-MIX were developed using eight high-yielding and/or high quality modern varieties. ENGCCP was the same as YQ-CCP, but grown in Hungary for three years before the experiment was started. This resulted in a genetic difference between the two CCPs as they were exposed to different environmental conditions. ELIT-CCP was developed in Hungary using seven elite wheat varieties (Mv-Béres, Mv-Süveges, Mv-Suba, Mv-Ködmön, MvVerbunkos, Mv-Csárdás and Mv-Magdaléna) having similar heading date, good yield, disease tolerance, high protein and gluten content and good breadmaking quality. HUNCCP was also developed in Hungary using old varieties and landraces resulted in early maturity and high plants $(>120 \mathrm{~cm})$. INRA-CCP was developed by crossing 60 parents ensuring high diversity in the population. MIX-AT was developed by equally mixing seeds of ten Austrian varieties (Lukullus, Blasius, Peppino, Pireneo, Stefanus, Arnold, Bitop, Capo, Midas and Tobias) in each year according to their TKW. Their quality parameters were discussed by Rakszegi et al. (2016). POP-AT was developed by mixing 18 cross-populations. Crosses included varieties such as Bitop (Austria), Mv-Kolo (Hungary), Pannonikus, Divana (Croatia) or Prairie Red (US). All the selected parental varieties had good disease resistance, good TKW, good tillering and not too short plant height. At the same time, three parents were high yielding while all the other varieties had very high quality. In spite of the careful selection of the varieties used for the development of CCPs/ mixtures, it could not necessarily be expected to reach high processing quality under organic or low-input conditions according to the previous studies (Döring et al. 2015; Murphy et al. 2005). This finding is also supported by the present study.

*Further details about the Electronic Supplementary Material (ESM) can be found at the end of the article. 


\section{Growing conditions}

Plants were grown at organic $(\mathrm{O})$ and 'low-input' $(\mathrm{LI})$ sites in five different countries [Austria (A), France (F), Hungary $(\mathrm{H})$, Switzerland $(\mathrm{CH})$ and the United Kingdom (UK)] in three different years (2011-2013) using a randomised complete block design (small plots) with 3 replicates to evaluate the stability of the quality parameters regarding cultivation with low external inputs. Austria and Hungary belong to the same geographical region, but all the five countries have different climatic conditions. Austria has a continental climate similarly to Hungary, but with oceanic effects. The Swiss site is in the continental environmental zone on a higher altitude with three times more precipitation than in Austria or Hungary. The UK site at East England has a temperate climate with regular rainfall, while the French location has mild winter. The weather conditions differed greatly between years. After a moderately dry first season in 2010/2011, the 2011/12 season brought an extreme drought (except in Switzerland and the UK) followed by an average season in 2012/13. In most cases the Hungarian locations got less precipitation and were warmer than the Austrian ones, and the difference was even greater when compared to the UK or Swiss weather conditions. In each countries the $\mathrm{O}$ and LI sites were located on neighbouring fields and the experiments were planted close to each other $(<1080 \mathrm{~m})$ to minimize confounding effects of differences in soil and climatic conditions. Herbicides, insecticides and artificial fertilizers were used in the 'low-input' fields when necessary, but fungicides were not (Table S2). Further growing and management parameters of two of the trial locations were detailed by Mikó et al. (2014).

\section{Physical properties}

The test weight (TW, g seed/100 litre) (MSZ 6367/4-1983, Foss Tecator 1241), the thousand-kernel weight (TKW, g/1000 kernels) (MSZ 6367/4-86), the kernel width and kernel length (Marvin Digital Seed Analyser) and the hardness index (HI) (AACC Method 55-31, Perten SKCS 4100) of the seeds were measured using samples from each of the three field replications.

\section{Compositional properties}

A $700 \mathrm{~g}$ of seeds per field replication were conditioned to $15.5 \%$ moisture content and milled by a Chopin CD1 Laboratory Mill to produce white flour. Wholemeal flour was produced by a Perten Laboratory Mill 3100. Crude protein content was analysed by the Kjeldahl method consistent with ICC method 105/2 using the Kjeltec 1035 Analyzer. Gluten content was measured by Glutomatic 2200 (ICC 137/1). Total and water extractable pentosans were determined using a colorimetric method as described by Douglas (1981). 


\section{Breadmaking properties}

Dough properties, such as water absorption, dough development time, dough stability and dough softening were measured by a Brabender Farinograph according to the standard ICC 115/1. Gluten index was calculated according to the ICC 155 standard method, while the gluten spread was measured according to a Hungarian standard [MSZ 6369/5-87 (1987)]. This parameter provides information about the proteolytic activity of flour by monitoring the changes in the diameter of a gluten ball after 1 hour at room temperature. Bread volume was estimated by the Zeleny sedimentation test (ICC 116/1, SediCom System).

\section{Statistical analyses}

GGE biplot analysis was carried out using GenStat 17.0 software (VSN International Ltd., Hemel Hemstead, UK), while one-way ANOVA and Tukey's post-hoc test were carried out using SPSS 16.0 software (SPSS Inc., Chicago, IL, USA).

GGE biplot illustrates the genotype plus genotype-by-environment variation using scores from a principal component analysis, but removing the environmental effects. The Ranking biplot [average-environment coordination (AEC) view of the GGE biplot] can be used to examine the performances of all genotypes within a specific environment. In the plot, the best performing and most stable genotypes are those whose projections onto the biplot axis are closest to the environment. The single-arrowed line is the AEC abscissa pointing to higher mean values of a given trait. The AEC ordinate points to greater variability (poorer stability) in both directions (Yan and Tinker 2006).

\section{Results}

Physical, compositional and processing quality of the CCPs and mixtures

Significant differences were found between the CCPs/mixtures in physical properties, such as test weight (TW), thousand-kernel weight (TKW) and hardness index (HI). The TW of NIAB-MIX and NIAB-CCP $(78 \mathrm{~kg} / \mathrm{hl})$ differed most significantly from that of the MIX-AT ( $84 \mathrm{~kg} / \mathrm{hl}$ ) (Table S1). The highest difference in TKW appeared between NIABMIX (36.1 g) and POP-AT (42.3 g), while INRA-CCP and NIAB-CCP had the softest kernels ( 43) compared to MIX-AT and POP-AT, which had the hardest kernels $(\sim 56)$. With regard to compositional properties, there was no significant difference between the $\mathrm{CCPs} /$ mixtures in protein content (13-14.7\%), but a large difference was found in the gluten content with NIAB-MIX and NIAB-CCP having the lowest ( $28 \%)$ and ELIT$\mathrm{CCP}$ the highest values (36\%). Some variation was also found in the starch content of the samples, but it was not significant (55.7-58.6\%). Dietary fibre content of the grains, such as TOT-AX and WE-AX varied more with ELIT-CCP having the highest $(20.3 \mathrm{mg} / \mathrm{g})$ and MIX-AT the lowest TOT-AX $(14.5 \mathrm{mg} / \mathrm{g})$. The mean WE-AX content was the greatest in ELIT-CCP $(7.6 \mathrm{mg} / \mathrm{g})$ and ENG-CCP $(8.3 \mathrm{mg} / \mathrm{g})$, while the lowest values were found in MIX-AT $(5.2 \mathrm{mg} / \mathrm{g})$ and YQ-CCP $(5.8 \mathrm{mg} / \mathrm{g})$. 
The compositional and physical properties of the CCPs/mixtures were compared in detail under the two management systems ( $\mathrm{O}$ and $\mathrm{LI})$ of three countries (A, H, UK). The management systems were found to have no significant effect on compositional parameters. Only the protein content differed significantly and only between the UKLI and UKO sites, where the protein content was $2 \%$ lower in each population at the organic site than at the low-input site (data not shown). In Austria and Hungary, the mean protein content of each population was higher than 14\%, where the HUN-CCP grown at the Hungarian sites was the most outstanding (Fig. S1). The lower protein content at the UK site might be resulted from the bigger seed size (TKW).

Greater variation was found in the AX content of the CCPs/mixtures, where ELITCCP, HUN-CCP and ENG-CCP had the highest TOT-AX and WE-AX content. CCPs/ mixtures developed in the UK or in Austria had lower dietary fibre content. The UK site differed from the $\mathrm{A}$ and $\mathrm{H}$ sites with a lower average $\mathrm{AX}$ content and with outstanding TOT-AX content in NIAB-CCP population (Fig. S1).

Processing quality of the grains was characterized by the gluten index (GI), the Zeleny sedimentation and the Farinograph quality number (Table S1). Based on these parameters, different quality groups could be distinguished. Three main groups could be separated with GI values of around 65 (HUN-CCP), 79 (NIAB-CCP, ELIT-CCP, INRA-CCP) and 97 (MIX-AT). Based on the Zeleny sedimentation, two main groups could be distinguished with sedimentation volume of around $22 \mathrm{ml}$ (INRA-CCP, YQ-CCP, NIAB-CCP, HUN-CCP) and $33 \mathrm{ml}$ (MIX-AT, POP-AT). The Farinograph quality number identified a group (MIX-AT and POP-AT) with very good baking quality (75), while NIAB-CCP and INRA-CCP showed the opposite value with quality numbers of 42.9 and 46.5 , respectively. These latter two populations and the YQ-CCP had the highest dough softening that indicates their high dough extensibility. The Farinograph dough stability was the highest for POP-AT (10.8 min), while the other CCPs/mixtures had a stability of around 3-4 min. The water absorption and dough development time are also important from a processing point of view. The dough development time of POP-AT was the slowest $(8.8 \mathrm{~min})$ while the dough of the other CCPs/mixtures developed much faster (around $2.5 \mathrm{~min}$ ). The lowest water absorption was found for NIAB-MIX and NIAB-CCP $(\sim 55 \%)$, while the highest was observed for POP-AT and ELIT-CCP ( 63\%).

Compared to the common control Mv-Emese, CCPs/mixtures had low TKW and kernel sizes, but based on the compositional properties, CCPs/mixtures could be competitive in respect of their quality, especially their protein content. Breadmaking quality data showed that CCPs/mixtures had smaller dough stability and higher dough extensibility than Mv-Emese. Although CCPs/mixtures were developed from the best performing varieties, they could only guarantee an average milling quality, but expected to keep a balance between good yield and good quality.

\section{Stability analysis of the CCPs/mixtures using GGE biplots and CV values}

In order to measure the stability and variability of the quality traits, the coefficient of variation (CV) was calculated, which is in negative correlation with stability (Table S3). 
Comparison of the $\mathrm{CV}$ values calculated for the organic and low input sites of the five countries showed that the CCPs/mixtures were more stable (having lower CV values) at low-input sites than at organic sites. The results for the ELIT-CCP, INRA-CCP, YQ-CCP and YQ-MIX were the most representative as they were grown in all five countries (A, F, $\mathrm{H}, \mathrm{CH}, \mathrm{UK}$ ). YQ-CCP and YQ-MIX had the most stable Farinograph quality (quality number and dough softening), while ELIT-CCP had consistently high stability for TOTAX content and water absorption at the low-input site.

A second group of populations (NIAB-CCP, NIAB-MIX and ENG-CCP) was grown in three countries (F, H, UK or A, CH, H; Table S1), and the NIAB-CCP was found to have stable Farinograph quality, protein and TOT-AX content at the low input site, while ENG-CCP had stable physical properties and WE-AX content at the organic site.

The third group of populations (HUN-CCP, MIX-AT and POP-AT) was grown only in two countries $(\mathrm{A}$ and $\mathrm{CH}$ ). These populations were found to be stable with respect to their compositional and breadmaking quality traits, especially at the low input sites. Physical properties of HUN-CCP and POP-AT were stable at the organic site, while MIX-AT had stable WE-AX content and GI at the organic sites (Table S3).

Stability of the physical, compositional and breadmaking quality properties was also assessed by GGE biplot analyses. According to the results, TW of MIX-AT, POP-AT, YQCCP, YQ-MIX, HUN-CCP and INRA-CCP was consistently high, as they are closer to the right side of the AEC abscissa (Fig. S2). At the same time, MIX-AT, POP-AT, YQCCP, YQ-MIX, HUN-CCP, INRA-CCP and ELIT-CCP had consistently high TKW as well. TW of NIAB-CCP and NIAB-MIX was lower than expected at ALI, AO and CHO sites [as CCPs/mixtures are located far from the end of the arrow/biplot origin/grand mean and stay opposite side of the AEC abscissa than the locations (ALI, AO, CHO)], but higher than expected at the HLI site (CCPs/mixtures and locations stay on the same side of the AEC abscissa). Interpreting in the same way, the TW of ENG-CCP and ELIT-CCP was lower than expected at the HLI site, but higher than expected at the $\mathrm{CHO}$ site.

TKW of NIAB-CCP, NIAB-MIX and ENG-CCP was lower than expected at the CHO site but higher than expected at the organic site of the UK and the low input sites of France and the UK. Although ENG-CCP had the highest mean TKW, its stability was low as it stays away from AEC abscissa. YQ-CCP and INRA-CCP had a mean TKW similar to the grand mean (end of arrow on AEC abscissa or biplot origin) (Fig. S2).

Stability of the compositional properties, such as the protein-, gluten-, TOT-AX and WE-AX content were also assessed. MIX-AT, POP-AT, YQ-CCP, YQ-MIX, HUN-CCP, INRA-CCP, ENG-CCP, ELIT-CCP and Mv-Emese had high and stable protein content, while MIX-AT, POP-AT, YQ-CCP, YQ-MIX, HUN-CCP, INRA-CCP and Mv-Emese had high and stable gluten content as well. The protein and gluten content of NIAB-CCP and NIAB-MIX were lower than expected at the CHO site, but higher than expected in Hungary (both at $\mathrm{O}$ and LI sites). The protein and gluten content of ENG-CCP and ELIT-CCP were higher than expected at the $\mathrm{CHO}$ site, but lower than expected at the HLI site (Fig. S3).

All the populations have higher than expected AX content at the Austrian organic site, while Mv-Emese had lower AX content at that site. In the same time, the fibre content of 
Mv-Emese was higher at the AO site than for any other CCPs/mixtures at any other sites, but it was very unstable. YQ-CCP and MIX-AT had the most stable and relatively high WE-AX content. ELIT-CCP had the highest mean TOT-AX and WE-AX content, while MIX-AT had a mean TOT-AX and WE-AX similar to the grand mean (Fig. S3).

Processing quality is affected not only by the protein content of wheat, but also by the dietary fibres. Arabinoxylans mostly affect the water absorption of the flour. According to our findings, the water absorption of the CCPs/mixtures was generally high and stable, with the exception of the ENG-CCP, NIAB-CCP and NIAB-MIX. The water absorption of NIAB-CCP and NIAB-MIX was lower than expected at the CHO site, but it was higher than expected at the UKO and HLI sites; however the opposite trend was observed for ENG-CCP (Fig. S4).

Dough quality was characterised by the gluten index (GI), the Zeleny sedimentation and the Farinograph parameters. Based on these parameters, the ELIT-CCP had the best performance with the highest and most stable mean values. Several other populations also had stable GI (POP-AT, YQ-CCP, YQ-MIX, INRA-CCP), Zeleny sedimentation (YQCCP, YQ-MIX, INRA-CCP) and Farinograph quality number (YQ-CCP, YQ-MIX, INRA-CCP, NIAB-MIX, NIAB-CCP) at most of the sites. At the same time, GI of HUNCCP, ENG-CCP and MIX-AT was lower than expected at the UKLI and UKO sites, and lower than expected for NIAB-MIX and NIAB-CCP at the CHO site. The Zeleny sedimentation of the NIAB populations was also lower than expected at the CHO site. HUNCCP, ENG-CCP and MIX-AT had lower, while POP-AT had higher Farinograph quality number than expected at the UKO site (Fig. S4).

\section{Discussion}

\section{Quality criteria}

Following the Hungarian wheat standard (MSZ6383:2012), based on the protein content, the ELIT-CCP, NIAB-MIX and POP-AT had premium quality, but the ELIT-CCP also had outstanding gluten content (36\%) and water absorption (64.3\%), while POP-AT had premium Farinograph quality number (78.4), water absorption (62.5\%) and dough stability (10.9 min) as well. The Farinograph quality of MIX-AT was also outstanding. Based on other parameters, the CCPs/mixtures were suitable for common milling purposes and bread production, although the Zeleny sedimentation was very low $(>30 \mathrm{ml})$ in most of the mixtures/CCPs. NIAB-CCP had poor Farinograph quality as well, meaning that this population is not suitable for bread production in Hungary. The Hungarian wheat standard was updated in 2012 by taking into consideration the internationally accepted quality criteria, but for some parameters it became stricter than those (Carson and Edwards 2009).

It is worth mentioning that CCPs/mixtures were studied at two to five locations in two to three years (Table S1), which could influence the results of the statistical analysis. In addition to the local weather conditions, previous crops, $\mathrm{N}$ fertilizer input or quantity of manure applied, were also different in the different countries and in the different years, 
which could also influence the performance and quality of the populations (Table S2). There are many studies going deep into these questions (e.g. Lammerts van Bueren and Myers 2012).

\section{Health}

Wheat and other cereals are major sources of dietary fibre (DF) in the human diet. Consumption of DF results in both lower cholesterol and glucose absorption in the small intestine and lower postprandial blood insulin levels (Moore et al. 1998; Lewis and Heaton 1999) related to reduced risk of diabetes type II. Apart from its nutritional relevance, DF is also important from a technological point of view as it strongly affects wheat functionality during cereal processing (Courtin and Delcour 2002; Frederix et al. 2004). According to Gebruers et al. (2008) and Ward et al. (2008), who studied the dietary fibre content of 150 wheat varieties with gas chromatography, the total arabinoxylan content could vary between 13.5 and $27.5 \mathrm{mg} / \mathrm{g}$ dry matter (dm) in flour and between 131 and 221 $\mathrm{mg} / \mathrm{g} \mathrm{dm}$ in bran. The water-extractable arabinoxylan (WE-AX) content of the seed could vary between 3 and $14 \mathrm{mg} / \mathrm{g} \mathrm{dm}$ in flour, but the $14 \mathrm{mg} / \mathrm{g}$ was an extremely outstanding value and was measured only in a Chinese variety. All the other varieties had less than 9 $\mathrm{mg} / \mathrm{g} \mathrm{dm} \mathrm{WE-AX} \mathrm{content.} \mathrm{In} \mathrm{our} \mathrm{study,} \mathrm{using} \mathrm{the} \mathrm{spectrophotometric} \mathrm{method} \mathrm{around}$ $10-11 \mathrm{mg} / \mathrm{g} \mathrm{dm}$ WE-AX content was measured in the flour of this Chinese variety, while the variety checks contained 4-7 mg/g dm WE-AX content. This means that the value of $8 \mathrm{mg} / \mathrm{g}$ could be stated as an outstanding value in our measurements, thus two populations (ELIT-CCP and ENG-CCP) were identified with high AX content. However, possible effects of the growing conditions should be taken into consideration, more than $50 \%$ of the variation in AX content could be attributed to the genotype (Gebruers et al. 2010), and therefore effective selection of the populations could be carried out targeting this trait.

\section{Stability}

No significant differences were found among the populations grown under low-input and organic management conditions in the physical, compositional or end-use properties, but there was a difference in the stability of these traits. The values of the coefficient of variation showed that the populations had more stable quality parameters at the low-input sites than at the organic sites. These values, together with the GGE biplot analysis, showed that the populations developed earlier by the breeders had higher stability than the populations (NIAB-CCP, NIAB-MIX) developed later in time (and tested for less years). Furthermore, some populations were found to be especially unstable at certain sites under organic growing conditions (mostly at $\mathrm{CHO}, \mathrm{UKO}$ or $\mathrm{AO}$ sites, depending on the trait). However, most of the populations showed good stability in their physical, compositional or end-use quality at all the other sites. Cultivar mixtures and composite cross-populations in cereal crops were found to stabilize yield in most studies (e.g. Dubin and Wolfe 1994; Mundt 2002). In addition, increased diversity in plants was often linked to higher productivity (Cardinale et al. 2011). In our study, the stability (CV) of the quality traits 
measured for CCPs/mixtures were mostly higher than that of the control variety, Mv-Emese, which refers to the quality stabilizing effect of the diversity found in mixtures or CCPs.

Genetic diversity present in the CCPs and mixtures could not necessarily able to keep the high processing quality of the parents under organic or low-input conditions, but at least a few of their end-use parameters could stay outstanding even under these new circumstances. It was also concluded that the diversity found in the mixtures and CCPs has stabilizing effect on the quality parameters, but a higher stability was observed under low-input than at organic conditions, which is in line with the different levels of external inputs even between these two management systems. Health related benefits (e.g. high fibre content) of CCPs and mixtures were also identified without resulting in significant reduction of their processing quality. These results could be useful not only for breeders but also for the consumers in the long run.

\section{Acknowledgements}

This research received funding from the European Community's Seventh Framework Programme (FP7/2007-2013) under the Grant Agreement n²45058-SOLIBAM (supplementary Hungarian project EU_BONUS_12-1-2012-0032).

\section{References}

Altieri, M.A. 1999. The ecological role of biodiversity in agroecosystems. Agriculture, Ecosystems and Environ. 74:19-31.

Cardinale, B.J., Matulich, K.L., Hooper, D.U., Byrnes, J.E., Duffy, E., Gamfeldt, L., Balvanera, P., O’Connor, M.I., Gonzales, A. 2011. The functional role of producer diversity in ecosystems. Am. J. Bot. 98:572-592.

Carson, G.R., Edwards, N.M. 2009. Criteria of wheat and flour quality. In: Khan, K., Shewry, P.R. (eds), Wheat: Chemistry and Technology. AACC International, Inc. St. Paul, MN, USA. pp. 97-118.

Ceccarelli, S., Grando, S., Baum, M. 2007. Participatory plant breeding in water-limited environments. Exp. Agric. 43:411-435.

Courtin, C.M., Delcour, J.A. 2002. Arabinoxylans and endoxylanases in wheat flour breadmaking. J. Cereal Sci. 35:225-243.

Döring, T.F., Annicchiarico, P., Clarke, S., Haigh, Z., Jones, H.E., Pearce, H., Snape, J., Zhan, J., Wolfe, M.S. 2015. Comparative analysis of performance and stability among composite cross populations, variety mixtures and pure lines of winter wheat in organic and conventional cropping systems. Field Crops Res. 183:235-245.

Douglas, S.G. 1981. A rapid method for the determination of pentosans in wheat flour. Food Chem. 7:139-145.

Dubin, H.J., Wolfe, M.S. 1994. Comparative behaviour of three wheat cultivars and their mixtures in India, Nepal and Pakistan. Field Crops Res. 39:71-83.

Finckh, M.R. 2008. Integration of breeding and technology into diversification strategies for disease control in modern agriculture. Eur. J. Plant Pathol. 121:399-409.

Frederix, S.A., Van Hoeymissen, K., Courtin, C.M., Delcour, J.A. 2004. Water-extractable and water unextractable arabinoxylans affect gluten agglomeration behaviour during wheat flour gluten-starch separation. J. of Agric. and Food Chem. 52:7950-7956.

Frison, E.A., Cherfas, J., Hodgkin, T. 2011. Agricultural biodiversity is essential for a sustainable improvement in food and nutrition security. Sustainability 3:238-253. 
Gebruers, K., Dornez, E., Boros, D., Fraś, A., Dynkowska, W., Bedo, Z., Rakszegi, M., Delcour, J.A., Courtin, C.M. 2008. Variation in the content of dietary fiber and components thereof in wheats in the HEALTHGRAIN Diversity Screen. J. of Agric. and Food Chem. 56:9740-9749.

Gebruers, K., Dornez, E., Bedo, Z., Rakszegi, M., Fras, A., Boros, D., Courtin, C.M., Delcour, J.A. 2010. Environment and genotype effect on the content of dietary fibre and its components in wheat in the HEALTHGRAIN diversity screen. J. of Agric. and Food Chem. 58:9353-9361.

Hajjar, R., Jarvis, D.I., Gemmill-Herren, B. 2008. The utility of crop genetic diversity in maintaining ecosystem services. Agric., Ecosystems and Environ. 123:261-270.

Heal, G., Walker, B., Levin, S., Arrow, K., Dasgupta, P., Daily, G., Ehrlich, P. 2004. Genetic diversity and interdependent crop choices in agriculture. Resource and Energy Economics 26:175-184.

Lammerts van Bueren, E.T., Jones, S.S., Tamm, L., Murphy, K.M., Myers, J.R., Leifert, C., Messmer, M.M. 2010. The need to breed crop varieties suitable for organic farming, using wheat, tomato and broccoli examples: A review. NJAS Wageningen J. of Life Sci. 58:193-205.

Lammerts van Bueren, E.T., Myers, J. 2012. Organic Crop Breeding. First Ed. John Wiley and Sons Inc. http:// onlinelibrary.wiley.com/doi/10.1002/9781119945932.fmatter/pdf

Lewis, S.J., Heaton, K.W. 1999. The metabolic consequences of slow colonic transit. Am. J. Gastroenterol. 94:2010-2016.

Mikó, P., Löschenberger, F., Hiltbrunner, J., Aebi, R., Megyeri, M., Kovács, G., Molnár-Láng, M., Vida, Gy., Rakszegi, M. 2014. Comparison of bread wheat varieties with different breeding origin under organic and low input management. Euphytica 199:69-80.

Moore, M.A., Beom Park, C., Tsuda, H. 1998. Soluble and insoluble fibre influences on cancer development. Crit. Rev. Oncol. Hematol. 27:229-242.

Mundt, C.C. 2002. Performance of wheat cultivars and cultivar mixtures in the presence of Cephalosporium stripe. Crop Protection 21:93-99.

Murphy, K., Lammer, D., Lyon, S., Carter, B., Jones, S.S. 2005. Breeding for organic and low-input farming systems: An evolutionary-participatory breeding method for in bred cereal grains. Renewable Agric. and Food Systems 20:48-55.

Newton, A.C., Begg, G.S., Swanston, J.S. 2009. Deployment of diversity for enhanced crop function. Ann. Appl. Biol. 154:309-322.

Østergård, H., Finckh, M.R., Fontaine, L., Goldringer, I., Hoad, S.P., Kristensen, K., Lammerts van Bueren, E.T., Mascher, F., Munk, L., Wolfe, M.S. 2009. Time for a shift in crop production: Embracing complexity through diversity at all levels. J. of Agric. and Food Information 89:1439-1445.

Phillips, S.L., Wolfe, M.S. 2005. Evolutionary plant breeding for low-input systems. J. of Agric. Sci. 143:245254.

Rakszegi, M., Mikó, P., Löschenberger, F., Hiltbrunner, J., Aebi, R., Knapp, S., Bede, K., Megyeri, M., Kovács, G., Molnár L.M., Vida, Gy., Láng, L., Bedő, Z. 2016. Comparison of quality parameters of wheat varieties with different breeding origin under organic and conventional conditions. J. of Cereal Sci. 69:297-305.

Suneson, C.A. 1956. An evolutionary plant breeding method. Agronomy J. 48:188-191.

Ward, J.L., Poutanen, K., Gebruers, K., Piironen, V., Lampi, A.M., Nyström, L., Andersson, A.A.M., Aman, P., Boros, D., Rakszegi, M., Bedo, Z., Shewry, P.R. 2008. The HEALTHGRAIN Cereal Diversity Screen: concept, results, and prospects. J. of Agric. and Food Chem. 56:9699-9709.

Wolfe, M.S., Baresel, J.P., Desclaux, D., Goldringer, I., Hoad, S., Kovacs, G., Löschenberger, F., Miedaner, T., Østergård, H., Lammerts van Bueren, E.T. 2008. Developments in breeding cereals for organic agriculture. Euphytica 163:323-346.

Yan, W., Tinker, N.A. 2006. Biplot analysis of multi-environment trial data: Principles and applications. Canad. J. of Plant Sci. 86:623-645. 


\section{Electronic Supplementary Material (ESM)}

Electronic Supplementary Material (ESM) associated with this article can be found at the website of CRC at http://www.akademiai.com/content/120427/

Electronic Supplementary Table S1. Mean and standard deviation (SD) of quality components of 10 wheat populations and their control (Mv-Emese) examined at organic and low input sites in 5 countries (A - Austria, H - Hungary, UK - United Kingdom, CH - Switzerland, F - France) for 3 years (2011-2013)

Electronic Supplementary Table S2. Main growing conditions and management parameters at organic and low input fields, where 10 winter wheat populations (ELIT-CCP, INRA-CCP, YQ-CCP, YQ-MIX, NIAB-CCP, NIAB-MIX, ENG-CCP, HUN-CCP, MIX-AT and POP-AT) and their control (Mv-Emese) were examined in five countries (A - Austria, H - Hungary, UK - United Kingdom, CH - Switzerland, F - France) for three growing seasons (2011-2013)

Electronic Supplementary Table S3. Coefficient of variation (CV) values for physical, compositional and breadmaking quality traits in 2 different management (M) systems (LI - low input, $\mathrm{O}$ - organic) based on the examination of 10 wheat populations and their control (Mv-Emese) in 5 countries (A - Austria, $\mathrm{H}$ - Hungary, UK - United Kingdom, CH - Switzerland, F - France) for 3 years (2011-2013)

Electronic Supplementary Figure S1. Compositional properties (a. protein content, b. total arabinoxylan - TOTAX content, c. water extractable arabinoxylan - WE-AX content, d. thousand-kernel weight - TKW) of 10 wheat populations and their control (Mv-Emese) examined in 3 years (2011-2013), 3 countries (A - Austria, $\mathrm{H}$ - Hungary, UK - United Kingdom) and 2 management systems (organic and low input)

Electronic Supplementary Figure S2. GGE biplot analysis based on the physical properties of 10 wheat populations (ELIT-CCP, INRA-CCP, YQ-CCP, YQ-MIX, NIAB-CCP, NIAB-MIX, ENG-CCP, HUN-CCP, MIX-AT and POP-AT) and their control (Mv-Emese) examined in 3 years (2011-2013), 5 countries (A - Austria, H Hungary, UK - United Kingdom, CH - Switzerland, F - France) and 2 management systems ( $\mathrm{O}$ - organic, LI - low input)

Electronic Supplementary Figure S3. GGE biplot analysis based on the compositional properties (protein and gluten content, total arabinoxylan - TOT-AX content, water extractable arabinoxylan - WE-AX content) of 10 wheat populations (ELIT-CCP, INRA-CCP, YQ-CCP, YQ-MIX, NIAB-CCP, NIAB-MIX, ENG-CCP, HUNCCP, MIX-AT and POP-AT) and their control (Mv-Emese) examined in 3 years (2011-2013), 5 countries (A - Austria, H - Hungary, UK - United Kingdom, CH - Switzerland, F - France) and 2 management systems (O - organic, $\mathrm{LI}$ - low input)

Electronic Supplementary Figure S4. GGE biplot analysis based on the breadmaking quality traits of 10 wheat populations (ELIT-CCP, INRA-CCP, YQ-CCP, YQ-MIX, NIAB-CCP, NIAB-MIX, ENG-CCP, HUN-CCP, MIX-AT and POP-AT) and their control (Mv-Emese) examined in 3 years (2011-2013), 5 countries (A Austria, H - Hungary, UK - United Kingdom, CH - Switzerland, F - France) and 2 management systems (O - organic, LI - low input) 